\title{
Sitzung vom 26. November 1894
}

\author{
Vorsitzender: Hr. E. Fischer, Präsident:
}

Das Protocoll der letzten Sitzung wird genehmigt.

Der Vorsitzende theilt mit, dass der Gesellschaft zur Enthüllungsfeier des Denkmals für Eilhard Mitscherlich am 1. December 1894 von Seiten des Comités eine Anzahl Zulasskarten übersandt worden ist, die er den Mitgliedern zur Verfügung stellt.

Sodann macht er der Gesellschaft die Mittheilung, dass auf Veranlassung der Vorstände der physikalischen und physiologischen Gesellschaft zu Berlin eine Anzahl wissenschaftlicher Vereine zusammengetreten sei, um eine Gedächtnissfeier für Hermann von Helmholtz zu veranstalten. Auf Befehl Seiner Majestät des Kaisers findet die Feier am Freitag, den 14. December 1894 in der Singakademie statt; sie soll in einer musikalischen Aufführung und einer Gedächtnissrede, gehalten von Hrn. Geheimrat von Bezold, bestehen.

Bei der Beschränktheit des Raumes können den einzelnen Vereinen nur eine geringe Anzahl von Einlasskarten zur Verfügung gestellt werden, die nur für Herren Gültigkeit haben. Entsprechend der Zahl der Berliner Mirglieder fallen der Deutschen chemischen Gesellschaft 40 Karten zu.

Die Vertheilung derselben innerhalb der Gesellschaft soll so erfolgen, dass jedem Mitgliede des Vorstandes je eine Karte und die übrig bleibenden denjenigen Berliner Mitgliedern, welche am längsten der Gesellschaft angehören, zugesandt werden. Diejenigen Herren, welche von den Karten keinen Gebrauch machen wollen, sollen gebeten werden, sie an das Secretariat der Gesellschaft zurückgelangen zu lassen.

Zu ausserordentlichen Mitgliedern werden proclamirt die Herren:

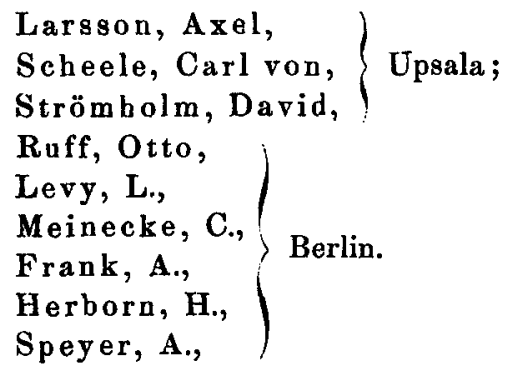


Za ausserordentlichen Mitgliedern werden vorgeschlagen die Herren:

Aubert, Axel, \} Spreestr. 1, Charlottenburg (durch E. Dedichen, Jens, $\}$ Täuber und M. Schöpff);

Leach, Mary F., Mount Holyoke College, South Hadley, Mass. (durch P. C. Freer und F. G. Novy);

Flatau, J., Berlinerstr. 131 II, Charlottenburg (durch C.

Liebermann and A. Bistraycki);

Baur-Breitenfeld, Karl von, Obere Karlstr. 1, Erlangen

Dietz, Karl, Südliche Stadtmauer 22, (durch

Becker, Julius, Harrenstr. 22,

Melzer, Herm., Halbmondstr. i. d. Oppelei, und Otto

Fuchs, Albert, Südliche Stadtmaner 14, Fischer);

Frankl, J., Kantstr. 160 pt., Charlottenburg (durch C. Liebermann und A. Bistrzycki);

Thiele, Herm. P. K. H., Lindenaustr. 39 III, Dresden (durch

W. Hempel und R. Möhlau);

Caroselli, Dr. Arnold,

Gurwitsch, Leo,

Hensch, Robert,

Zündel, Charles,

Jedlicka, Johann,

Messing, Stanislaus,

Chemie-
Schule, $\left(\begin{array}{c}\text { Mülhausen i. E. } \\ \text { (durch E. Noelting } \\ \text { und E. Wild); }\end{array}\right.$

Meyer, Alfred, Lutterbacherstr. 17,

Gassmann, Carl, Judengasse,

Moskowitz, N., Englische Str. 23 c., Haus 8 II, Charlottenburg (durch C. Liebermann und C. Maull).

Für die Bibliothek sind als Geschenk eingegangen:

193. Relatorio annual do instituto agronomico do estado de São Paulo (Brazil) em Campinas 1893; pelo Director F. W. Dafert. S. Paulo 1894.

751. Gattermann, Ludwig. Die Praxis des organischen Chemikers. Leipzig 1894.

Der Vorsitzende:

Der Schriftfährer:

E. Fischer.

I. $\mathbf{v}$.

W. Will. 